\title{
A search for Galactic transients disguised as gamma-ray bursts
}

\author{
A. Clerici ${ }^{1}$, C. Guidorzi ${ }^{1}$, and V. La Parola ${ }^{2}$ \\ 1 Department of Physics and Earth Sciences, University of Ferrara, via Saragat 1, 44122 Ferrara, Italy \\ e-mail: aurora.clerici@student.unife.it \\ 2 INAF-Istituto di Astrofisica Spaziale e Fisica Cosmica di Palermo, via U. La Malfa 153, 90146 Palermo, Italy
}

Received 18 June 2015 / Accepted 19 August 2015

\begin{abstract}
Context. A significant fraction of cosmological gamma-ray bursts (GRBs) are characterised by a fast rise and exponential decay (FRED) temporal structure. This is not a distinctive feature of this class, since it is observed in many Galactic transients and is likely descriptive of a sudden release of energy followed by a diffusion process. Possible evidence has recently been reported for a Galactic contamination in the sample of FRED GRBs discovered with Swift.

Aims. We searched for possible Galactic intruders disguised as FRED GRBs in the Swift catalogue up to September 2014.

Methods. We selected 181 FRED GRBs (2/3 with unknown redshift) and considered different subsamples. We tested the degree of isotropy through the dipole and quadrupole moment distributions, both with reference to the Galaxy and independent of any coordinate system as well as with the two-point angular autocovariance function. In addition, we searched for possible indicators of a Galactic origin among the spectral and temporal properties of individual GRBs.

Results. We found marginal $(\sim 3 \sigma)$ evidence for an excess of FREDs with unknown redshift towards the Galactic plane compared with what is expected for an isotropic distribution corrected for the non-uniform sky exposure. However, when we account for the observational bias against optical follow-up observations of low-Galactic latitude GRBs, the evidence for anisotropy decreases to $\sim 2 \sigma$. In addition, we found no statistical evidence for different spectral or temporal properties from the bulk of cosmological GRBs. Conclusions. We found marginal evidence for the presence of a disguised Galactic population among Swift GRBs with unknown redshift. The estimated fraction is $f=(19 \pm 11) \%$, with an upper limit of $34 \%$ (90\% confidence).
\end{abstract}

Key words. gamma-ray burst: general - methods: statistical

\section{Introduction}

Gamma-ray bursts (GRBs) are known to be cosmological transient sources that, for long duration GRBs, signal the very final evolutionary stage of a type of massive star and, for short duration GRBs, likely signal the act of merging of a compact binary system (see Mészáros \& Gehrels 2012 for a review). Owing to their extreme luminosity, GRBs are routinely detected from cosmological distances up to redshifts $z \sim 9.4$ (Cucchiara et al. 2011). The consequent highly isotropic sky distribution was interpreted as evidence for a cosmological origin prior to the first redshift measurements (Hartmann \& Epstein 1989; Hartmann \& Blumenthal 1989; Briggs et al. 1996). Once the cosmological nature of long duration GRBs was finally established, a number of studies reported evidence for anisotropy in the distribution of short duration GRBs (Balazs et al. 1998; Magliocchetti et al. 2003; Vavrek et al. 2008; but see also Bernui et al. 2008), or evidence for a correlation between short GRBs and galaxies in the local Universe (Tanvir et al. 2005).

Recently, Tello et al. (2012; hereafter T12) exploited the real-time, arcmin-sized localisation capabilities of the Swift Burst Alert Telescope (BAT; Barthelmy et al. 2005) to test the isotropy of a special class of long GRBs, the so-called fast-rise exponential-decay (FRED) GRBs. Not only is their time profile very common among GRBs (Norris et al. 1996), but it is also descriptive of various high-energy outbursts from Galactic sources, such as X-ray binaries (Remillard \& McClintock 2006), X-ray bursters (Lewin et al. 1993), magnetars (Mereghetti 2008), as well as unidentified Galactic transients (Kasliwal et al. 2008; Castro-Tirado et al. 2008; Stefanescu et al. 2008). This kind of universality is observed across different wavelengths all the way to the radio bands, where analogous flares are observed over timescales, from seconds or less than seconds up to years, due to a broad range of astrophysical sources (Pietka et al. 2015). The ubiquity of FRED-like transient profiles is explained as descriptive of a sudden energy release, followed by some cooling and diffusion process. The T12 study found some evidence $(<3 \sigma$ confidence) for a deviation from isotropy of the sky distribution of Swift FREDs with unknown $z$. They attribute this deviation to the presence of Galactic unidentified sources, which would make up to $\sim 27 \%$ of the observed unknown- $z$ FRED population.

Motivated by their results, we carried out a similar investigation on an updated sample of the Swift FRED catalogue, adopting independent selection criteria. We analysed the degree of isotropy both with reference to the Galactic plane and via a search, which is independent of a reference system. In addition, to further characterise the nature of the Galactic candidates, we examined on a statistical basis the spectral and temporal properties of the $\gamma$-ray prompt and X-ray afterglow emission as observed with Swift BAT and X-ray Telescope (XRT; Burrows et al. 2005) and compared these properties with a sample of cosmological GRBs. The paper is organised as follows: the sample selection and data analysis are described in Sects. 2 and 3, respectively. Section 4 presents the results, which are discussed in Sect. 5. 


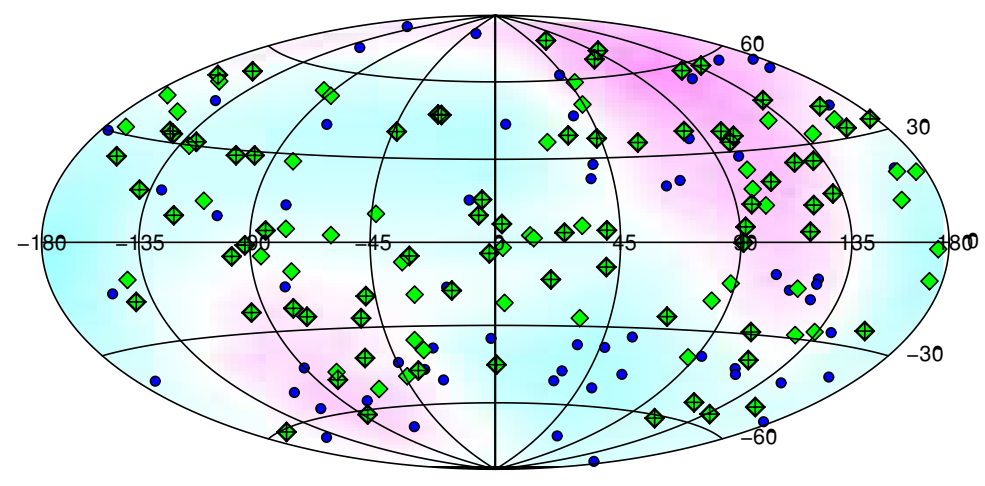

Fig. 1. Exposure map of BAT in Galactic coordinates from January 2005 to September 2014. Green diamonds (blue circles) are all FREDs with unknown (known) $z$. They jointly constitute S1. All green diamonds are sample S2. Crossed green diamonds are those unknown$z$ FREDs with a unique detected peak (sample S3). The three sets include 181, 119, and 71 GRBs, respectively.

Table 1. GRB sample sizes.

\begin{tabular}{llrr}
\hline \hline Sample & Description & $\begin{array}{r}\text { Size } \\
\text { (this work) }\end{array}$ & $\begin{array}{r}\text { Size } \\
\text { (T12) }\end{array}$ \\
\hline S1 & all FREDs & 181 & 111 \\
S2 & all FREDs, no $z$ & 119 & 77 \\
S3 & all FREDs, no $z, 1$ peak & 71 & 49 \\
STot & all GRBs & 904 & - \\
SnFnZ & all non-FREDs, no $z$ & 506 & - \\
\hline
\end{tabular}

\section{Sample selection}

Starting from a sample of 904 GRBs detected by Swift-BAT in the time interval January 2005 to September 2014, we selected those that had explicitly been tagged by the BAT team as FREDs in the GCN circulars. We ended up with 181 FREDs out of 904 GRBs, whereas T12 found 111 FREDs out of 596 GRBs. We further characterised the number of peaks of each GRB by means of the MEPSA code (Guidorzi 2015), capable of identifying relatively faint peaks at very different timescales. We set a threshold of 4.5 on the minimum signal-to-noise as yielded by MEPSA, which ensures a negligible false positive rate for our light curve sample. We then considered three different samples: i) the full sample; ii) the subsample of GRBs with unknown redshift $z$; iii) the subsample of GRBs with unknown $z$ and with a single peak detected with MEPSA. Hereafter these samples are referred to as S1, S2, and S3, including 181, 119, and 71 GRBs, respectively. Their sky distributions in Galactic coordinates are shown in Fig. 1. To assess the role of observational biases, for comparison we also consider two additional samples detected in the same time interval: (i) all 904 BAT-discovered GRBs, and (ii) the complementary sample of 506 non-FREDs GRBs with unknown $z$. Hereafter, we refer to them as STot and SnFnZ, respectively. Table 1 summarises the samples' sizes and the corresponding values of T12.

\section{Data analysis}

For each real sample, we calculated the three Galactic dipole moments $(\sin b, \cos b \sin l, \cos b \cos l)$, and the five quadrupole moments $\left(\sin ^{2} b-1 / 3, \cos ^{2} b \sin 2 l, \cos ^{2} b \cos 2 l, \sin 2 b \sin l\right.$, $\sin 2 b \cos l$ ) with an emphasis on those related to the Galactic plane, i.e. $\sin b$ and $\left(\sin ^{2} b-1 / 3\right)$. We also calculated the somewhat redundant equatorial dipole $\sin \delta$ and quadrupole $\left(\sin ^{2} \delta-\right.$ $1 / 3$ ) moments to better evaluate possible anisotropies connected with observational biases. In addition, we calculated the twopoint angular correlation function (Hartmann \& Blumenthal 1989). We also carried out a search, which is independent of a reference system, for anisotropies that are possibly unrelated with our Galaxy by evaluating Rayleigh-Watson $\mathcal{W}$ and Bingham $\mathcal{B}$ statistics (Briggs 1993). Everything was then compared with the expectations for isotropy following two independent approaches: Monte Carlo simulations and sky pixelisation.

The exposure map of Swift-BAT (Fig. 1) was obtained from the limiting flux map for the considered time interval, processing the BAT data with the Bat_Imager software (Segreto et al. 2010), and following the procedure described in Cusumano et al. (2010).

\subsection{Monte Carlo simulations (method 1)}

For each sample, we generated $10^{3}$ synthetic samples, each having as many positions as the corresponding real sample. Each fake position was generated according to the following procedure: i) a random position is drawn from an isotropic distribution; ii) the position is accepted or rejected, depending on the outcome of a binomial variate, whose acceptance probability was set to the fractional exposure of that position. The procedure ends as soon as the desired number of positions is achieved.

\subsection{Sky pixelisation (method 2)}

The entire sky was split into a homogeneous grid of 5292 pixels, following the technique described by Tegmark (1996), with angular resolution of 3.2 . Each pixel was then assigned the corresponding fractional exposure value. The choice of the angular resolution was driven by the need to ensure an adequate coverage of the BAT exposure map. The expected moments were calculated over the entire sky grid by weighting the contribution of each sky pixel by the associated fractional exposure. The uncertainties affecting the observed moments were calculated from the expected variances in the Gaussian limit ensured by the central limit theorem due to $N \gg 1$ events: $\sigma^{2}(\langle\sin b\rangle)=1 /(3 N)$, $\sigma^{2}\left(\left\langle\sin ^{2} b\right\rangle\right)=4 /(45 N)$ for the $\left(\sin ^{2} b-1 / 3\right)$ term, and $\sigma^{2}=$ $4 /(15 N)$ for the other quadrupole terms (Briggs 1993).

\section{Results}

\subsection{Dipole and quadrupole moments}

Table 2 reports the observed moments along with the expected values and intervals. While none of the dipole moments deviate by $>2 \sigma$ from isotropy, the Galactic quadrupole moment of S2 is marginally $(-2.8 \sigma)$ lower than the isotropic-expected Galactic quadrupole moment. An analogous result is obtained for $\mathrm{SnFnZ}$ 
A. Clerici et al.: A search for Galactic transients disguised as gamma-ray bursts

Table 2. Average dipolar and quadrupolar moments for the different samples.

\begin{tabular}{|c|c|c|c|c|c|c|}
\hline Sample & Moment & Observed & $\begin{array}{r}\text { Expected }^{a} \\
\text { (sky pix.; } \pm 1 \sigma)\end{array}$ & $\begin{array}{c}\text { Discrepancy } \\
(\sigma)\end{array}$ & \multicolumn{2}{|c|}{ Expected interval (MC) ${ }^{b}$} \\
\hline $\begin{array}{l}\text { S1 } \\
\text { S1 } \\
\text { S1 } \\
\text { S1 } \\
\text { S1 }\end{array}$ & $\begin{array}{c}\langle\cos b \cos l\rangle \\
\langle\sin b\rangle \\
\langle\sin \delta\rangle \\
\left\langle\sin ^{2} b-1 / 3\right\rangle \\
\left\langle\sin ^{2} \delta-1 / 3\right\rangle\end{array}$ & $\begin{array}{r}-0.010 \\
0.028 \\
0.065 \\
-0.009 \\
0.074 \\
\end{array}$ & $\begin{array}{r}-0.010 \pm 0.043 \\
0.022 \pm 0.043 \\
0.035 \pm 0.043 \\
0.014 \pm 0.022 \\
0.039 \pm 0.022\end{array}$ & $\begin{array}{c}0.0 \\
+0.1 \\
+0.7 \\
-1.0 \\
+1.5\end{array}$ & $\begin{array}{l}-0.050,+0.031 \\
-0.018,+0.064 \\
-0.012,+0.074 \\
-0.009,+0.035 \\
+0.016,+0.062\end{array}$ & $\begin{array}{l}-0.091,+0.072 \\
-0.061,+0.105 \\
-0.052,+0.120 \\
-0.030,+0.057 \\
-0.005,+0.085\end{array}$ \\
\hline $\begin{array}{l}\mathrm{S} 2 \\
\mathrm{~S} 2 \\
\mathrm{~S} 2 \\
\mathrm{~S} 2 \\
\mathrm{~S} 2\end{array}$ & $\begin{array}{c}\langle\cos b \cos l\rangle \\
\langle\sin b\rangle \\
\langle\sin \delta\rangle \\
\left\langle\sin ^{2} b-1 / 3\right\rangle \\
\left\langle\sin ^{2} \delta-1 / 3\right\rangle\end{array}$ & $\begin{array}{r}-0.005 \\
0.097 \\
0.070 \\
-0.063 \\
0.103 \\
\end{array}$ & $\begin{array}{r}-0.010 \pm 0.053 \\
0.022 \pm 0.053 \\
0.035 \pm 0.053 \\
0.014 \pm 0.027 \\
0.039 \pm 0.027\end{array}$ & $\begin{array}{l}+0.1 \\
+1.4 \\
+0.7 \\
-2.8 \\
+2.3 \\
\end{array}$ & $\begin{array}{l}-0.063,+0.036 \\
-0.030,+0.075 \\
-0.018,+0.086 \\
-0.012,+0.043 \\
+0.011,+0.068\end{array}$ & $\begin{array}{l}-0.108,+0.081 \\
-0.089,+0.125 \\
-0.072,+0.141 \\
-0.036,+0.072 \\
-0.016,+0.098\end{array}$ \\
\hline $\begin{array}{l}\mathrm{S} 2^{c} \\
\mathrm{~S} 2^{c} \\
\mathrm{~S} 2^{c} \\
\mathrm{~S} 2^{c} \\
\mathrm{~S} 2^{c}\end{array}$ & $\begin{array}{c}\langle\cos b \cos l\rangle \\
\langle\sin b\rangle \\
\langle\sin \delta\rangle \\
\left\langle\sin ^{2} b-1 / 3\right\rangle \\
\left\langle\sin ^{2} \delta-1 / 3\right\rangle\end{array}$ & $\begin{array}{r}-0.005 \\
0.097 \\
0.070 \\
-0.063 \\
0.103 \\
\end{array}$ & $\begin{array}{r}0.017 \pm 0.053 \\
-0.008 \pm 0.053 \\
-0.022 \pm 0.053 \\
-0.013 \pm 0.027 \\
0.056 \pm 0.027\end{array}$ & $\begin{array}{l}-0.4 \\
+2.0 \\
+1.7 \\
-1.8 \\
+1.7 \\
\end{array}$ & $\begin{array}{l}-0.036,+0.066 \\
-0.057,+0.043 \\
-0.079,+0.034 \\
-0.042,+0.015 \\
+0.031,+0.086\end{array}$ & $\begin{array}{l}-0.080,+0.123 \\
-0.108,+0.096 \\
-0.130,+0.085 \\
-0.069,+0.045 \\
-0.001,+0.114\end{array}$ \\
\hline $\begin{array}{l}\text { S3 } \\
\text { S3 } \\
\text { S3 } \\
\text { S3 } \\
\text { S3 }\end{array}$ & $\begin{array}{c}\langle\cos b \cos l\rangle \\
\langle\sin b\rangle \\
\langle\sin \delta\rangle \\
\left\langle\sin ^{2} b-1 / 3\right\rangle \\
\left\langle\sin ^{2} \delta-1 / 3\right\rangle\end{array}$ & $\begin{array}{r}-0.005 \\
0.111 \\
0.097 \\
-0.032 \\
0.102 \\
\end{array}$ & $\begin{array}{r}-0.010 \pm 0.068 \\
0.022 \pm 0.068 \\
0.035 \pm 0.068 \\
0.014 \pm 0.035 \\
0.039 \pm 0.035 \\
\end{array}$ & $\begin{array}{l}+0.1 \\
+1.3 \\
+0.9 \\
-1.3 \\
+1.8 \\
\end{array}$ & $\begin{array}{l}-0.080,+0.059 \\
-0.046,+0.088 \\
-0.038,+0.106 \\
-0.025,+0.048 \\
+0.004,+0.078 \\
\end{array}$ & $\begin{array}{l}-0.148,+0.117 \\
-0.109,+0.165 \\
-0.109,+0.171 \\
-0.059,+0.083 \\
-0.030,+0.121\end{array}$ \\
\hline $\begin{array}{l}\text { SnFnZ } \\
\text { SnFnZ } \\
\text { SnFnZ } \\
\text { SnFnZ } \\
\text { SnFnZ }\end{array}$ & $\begin{array}{c}\langle\cos b \cos l\rangle \\
\langle\sin b\rangle \\
\langle\sin \delta\rangle \\
\left\langle\sin ^{2} b-1 / 3\right\rangle \\
\left\langle\sin ^{2} \delta-1 / 3\right\rangle\end{array}$ & $\begin{array}{r}0.036 \\
0.011 \\
0.040 \\
-0.014 \\
0.046 \\
\end{array}$ & $\begin{array}{r}-0.010 \pm 0.026 \\
0.022 \pm 0.026 \\
0.035 \pm 0.026 \\
0.014 \pm 0.013 \\
0.039 \pm 0.013\end{array}$ & $\begin{array}{c}+1.8 \\
0.0 \\
+2.0 \\
-2.1 \\
+0.5\end{array}$ & $\begin{array}{l}- \\
- \\
- \\
- \\
- \\
\end{array}$ & $\begin{array}{l}- \\
- \\
- \\
- \\
-\end{array}$ \\
\hline $\begin{array}{l}\text { STot } \\
\text { STot } \\
\text { STot } \\
\text { STot } \\
\text { STot }\end{array}$ & $\begin{array}{c}\langle\cos b \cos l\rangle \\
\langle\sin b\rangle \\
\langle\sin \delta\rangle \\
\left\langle\sin ^{2} b-1 / 3\right\rangle \\
\left\langle\sin ^{2} \delta-1 / 3\right\rangle\end{array}$ & $\begin{array}{l}0.014 \\
0.011 \\
0.040 \\
0.017 \\
0.037 \\
\end{array}$ & $\begin{array}{r}-0.010 \pm 0.019 \\
0.022 \pm 0.019 \\
0.035 \pm 0.019 \\
0.014 \pm 0.010 \\
0.039 \pm 0.010\end{array}$ & $\begin{array}{l}+1.2 \\
-0.6 \\
+0.2 \\
+0.4 \\
-0.2\end{array}$ & $\begin{array}{l}- \\
- \\
- \\
- \\
-\end{array}$ & $\begin{array}{l}- \\
- \\
- \\
- \\
-\end{array}$ \\
\hline
\end{tabular}

Notes. The real value moments are reported and associated with the values obtained from the sky pixelisation method, along with their discrepancies in $\sigma$ units. The last two columns report the MC $1 \sigma$ and $2 \sigma$ (Gaussian-equivalent) confidence intervals. ${ }^{(a)}$ Estimated through the sky pixelisation (method 2) in the Gaussian limit ensured by the central limit theorem. ${ }^{(b)}$ Estimated through MC simulations (method 1). ${ }^{(c)}$ S2 is compared with expectations that modelled observational biases connected with the redshift measurement (Sect. 4.4).

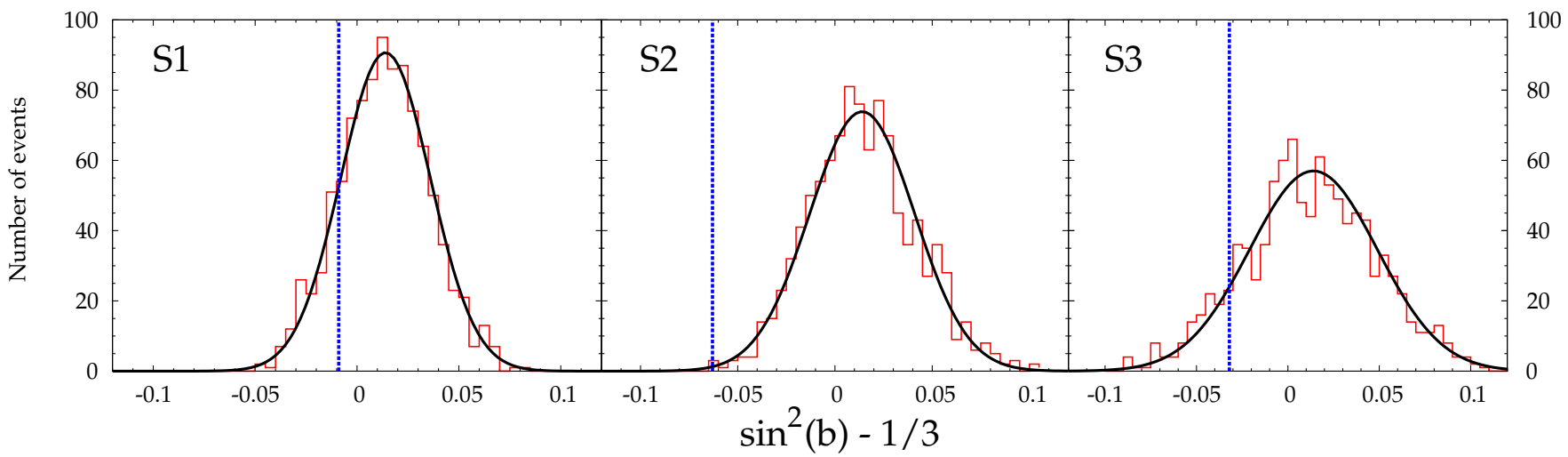

Fig. 2. Galactic quadrupole moment distributions obtained from MC simulations (method 1; histograms) and from the sky pixelisation in the Gaussian limit (method 2; solid curves) compared with the values observed in the real samples (vertical lines) for S1, S2, and S3 (left, middle, and right panels, respectively).

with a less significant deviation though $(-2.1 \sigma)$. This suggests that the unknown- $z$ FREDs tend to cluster at low Galactic latitudes, or equivalently, that they are rarely found at high latitudes. Among the other Galactic quadrupole moments, only $\sin 2 b \cos l$ exhibits two $>2 \sigma$ deviations from isotropy, $-2.5 \sigma$
$(-2.4 \sigma)$ for S1 (S2). Figure 2 shows the Galactic quadrupole moment $\left(\sin ^{2} b-1 / 3\right)$ for each of the three samples obtained with both methods assuming isotropy, corrected for the non-uniform exposure map, to be compared with the value observed in the corresponding real sample. 
Table 3. Observed values of Rayleigh-Watson $(\mathcal{W})$ and Bingham $(\mathcal{B})$ statistics and confidence intervals expected for an isotropic distribution corrected for non-uniform exposure.

\begin{tabular}{lcrrrrr}
\hline \hline Sample & Statistics & Observed & \multicolumn{3}{c}{$\begin{array}{c}\text { Expected interval }(\mathrm{MC})^{a} \\
(1 \sigma)\end{array}$} \\
& & \multicolumn{5}{c}{$(2 \sigma)$} \\
\hline S1 & $\mathcal{W}$ & 2.6 & 0.9 & 6.0 & 0.2 & 10.5 \\
S2 & $\mathcal{W}$ & 3.7 & 0.9 & 5.6 & 0.2 & 10.2 \\
S3 & $\mathcal{W}$ & 3.3 & 0.9 & 5.5 & 0.2 & 10.1 \\
\hline S1 & $\mathcal{B}$ & 14.2 & 4.9 & 16.0 & 1.7 & 24.8 \\
S2 & $\mathcal{B}$ & 19.4 & 3.7 & 13.8 & 1.6 & 21.5 \\
S3 & $\mathcal{B}$ & 9.8 & 2.9 & 11.1 & 1.1 & 18.6 \\
\hline
\end{tabular}

Notes. ${ }^{(a)}$ Estimated through MC simulations (method 1).

\section{2. $\mathcal{W}$ and $\mathcal{B}$ statistics}

Following Briggs (1993), for each position $\left(l_{i}, b_{i}\right)$ we considered the associated unit vector $\boldsymbol{r}_{i}=\left(x_{i}, y_{i}, z_{i}\right)$ and calculated the Rayleigh-Watson statistic defined as

$\mathcal{W}=\frac{3}{N}\left|\sum_{i=1}^{N} \boldsymbol{r}_{i}\right|^{2}$.

This statistic characterises the dipole moment and, in the absence of any preferred direction, is asymptotically distributed as $\chi_{3}^{2}$ for large $N$. The Bingham statistic $\mathcal{B}$ is defined as

$\mathcal{B}=\frac{15 N}{2} \sum_{k i=1}^{3}\left(\lambda_{k}-\frac{1}{3}\right)^{2}$,

where $\lambda_{k}$ are the eigenvalues of the so-called "orientation" matrix, which is equivalent to the quadrupole matrix and is defined as

$M_{N}=\frac{1}{N} \sum_{i=1}^{N}\left(\begin{array}{lll}x_{i} x_{i} & x_{i} y_{i} & x_{i} z_{i} \\ y_{i} x_{i} & y_{i} y_{i} & y_{i} z_{i} \\ z_{i} x_{i} & z_{i} y_{i} & z_{i} z_{i}\end{array}\right)$.

$\mathcal{B}$ measures the deviation of the eigenvalues from the value $1 / 3$ expected for isotropy and is asymptotically distributed as $\chi_{5}^{2}$. Because of the non-uniform sky exposure we only applied method 1 to derive the expected intervals for both statistics. Table 3 reports the observed values and corresponding intervals for isotropy. As for the Galactic moments, we did not find evidence for any preferred direction with a non-zero dipole moment, all within $1 \sigma$ confidence. As for the quadrupole term, although all of them lie within $2 \sigma$, S2 shows the largest deviation. In particular, it mildly suggests a clustering of unknown- $z$. FREDs along the Galactic direction $l=130^{\circ}, b=+13^{\circ}$ $\left(\alpha=39^{\circ}, \delta=+75^{\circ}, \mathrm{J} 2000\right)$ and its antipodal direction, i.e. close to celestial poles. From Table 2 the equatorial quadrupole moment $\left(\sin ^{2} \delta-1 / 3\right)$ only exceeds the expected isotropic value for S2 by $2.3 \sigma$, thus lending support to the enhanced presence of unknown- $z$ FREDs towards the celestial poles.

The unknown- $z$ non-FREDs GRBs (SnFnZ) show a similar behaviour: their Galactic quadrupole moment deviates by $-2.1 \sigma$ from isotropy. In contrast, in spite of the best statistical sensitivity, the whole BAT-detected sample (STot) shows no evidence at all for non-zero quadrupole moments.

\subsection{Two-point angular autocovariance}

Finally, we calculated the distribution of the two-point angular autocovariance function by taking all the pairs within a given

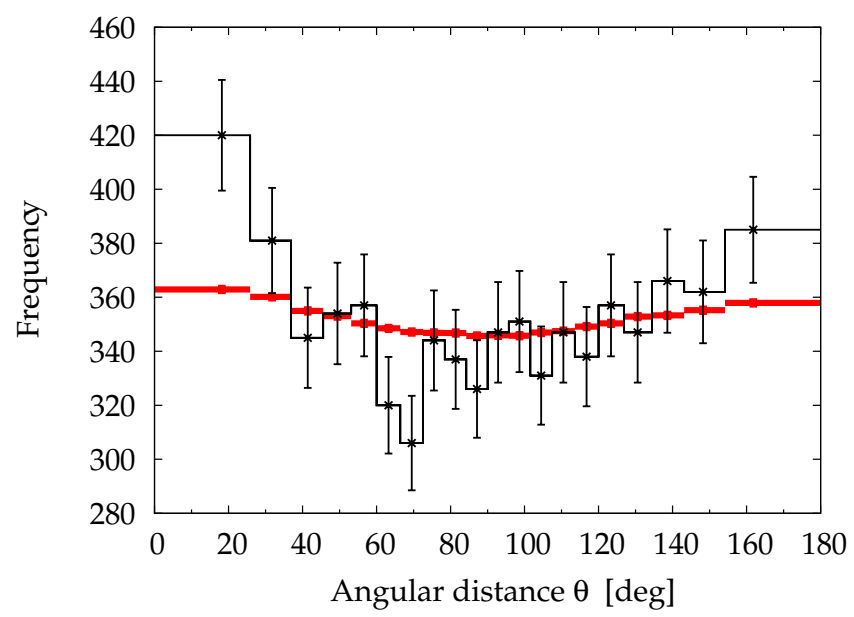

Fig. 3. Histogram of the angular autocovariance function distribution observed for S2 compared with what is expected taking the non-uniform sky exposure into account (thick red line). The bin width is uniform in $\cos \theta$.

sample. In particular, we considered $\cos \theta$ rather than $\theta$ (angular distance between a pair), given that for isotropy and uniform exposure the expected distribution is uniform in $\cos \theta$. Likewise, the expected distribution for isotropy was only derived through method 1 to account for the non-uniform exposure map. The most remarkable deviations are observed for $\mathrm{S} 2$, as shown in Fig. 3.

The thick line shows the isotropic-expected value as a function of the angular distance. The displayed uncertainties on the observed numbers are their square root values, under the assumption of Poisson statistics. Strictly speaking, this is not correct: the ensemble of $\theta$ values are not fully independent of each other, since the entire set of possible pairs is not independent. However, given the large number of pairs the degree of correlation is relatively small, and so, as a matter of fact, Poissonian uncertainties are realistic.

Unknown- $z$ FREDs seem to cluster on angular scales $<30^{\circ}$ (between $2 \sigma$ and $3 \sigma$ significance) at the expense of those in the range $60^{\circ}-90^{\circ}$. A $\chi^{2}$ test yields a p-value of $15 \%\left(\chi^{2} /\right.$ d.o.f. $=$ 25.4/19). However, this must be taken as a loose indication because of the lack of statistical independence of the different bins.

\subsection{Observational biases connected with redshift measurement}

In the light of the previous results, we tried to account for the observational biases involved in measuring $z$ that come into play when one considers the samples of unknown- $z$ GRBs with some evidence for anisotropy, S2 and SnFnZ. To this aim, using method 1 we generated another set of 1000 synthetic samples the same size as S2, which took the following two main sources of bias into account:

- Galactic dust extinction disfavours optical observations of low Galactic latitude GRBs;

- ground-based follow-up observations are less probable around the celestial poles (Fynbo et al. 2009).

To model the first bias, we considered the Galactic extinction as measured by Schlafly \& Finkbeiner (2011) in terms of $A_{V}{ }^{1}$. We

1 Available at http://irsa.ipac.caltech.edu/applications/ DUST/ 
A. Clerici et al.: A search for Galactic transients disguised as gamma-ray bursts

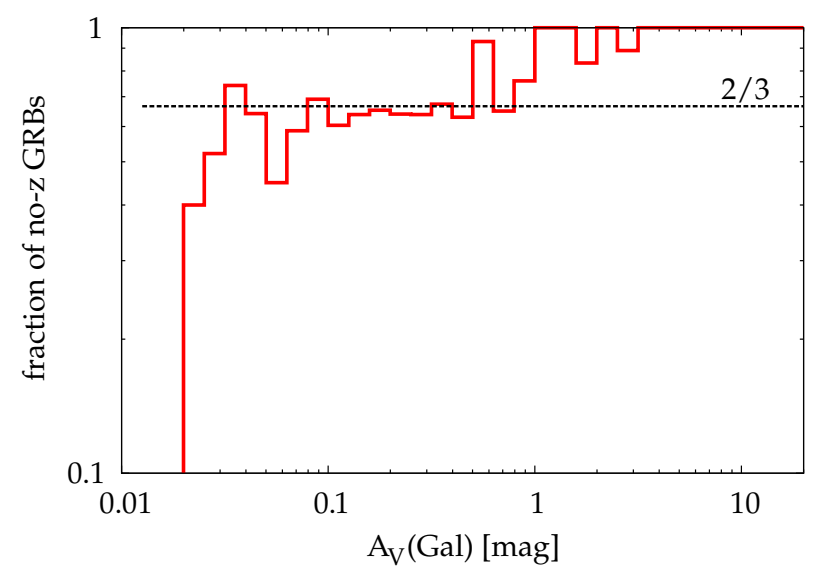

Fig. 4. Fraction of GRBs with unknown redshift as a function of Galactic dust extinction.

then obtained the $A_{V}(\mathrm{Gal})$ distribution for the positions of all the GRBs belonging to STot and split it into two groups, with and without measured $z$, respectively. For each $A_{V}(\mathrm{Gal})$ bin, we calculated the fraction of GRBs with unknown $z$ over the total. The result is shown in Fig. 4. As long as it is $A_{V}(\mathrm{Gal})<1 \mathrm{mag}$, the fraction is about $2 / 3$, i.e. the same value that is obtained over the entire Swift catalogue. However, for $A_{V}(\mathrm{Gal})>1$ mag almost all of the GRBs have no redshift. In generating the synthetic samples analogous to S2, each random position was accepted depending on the outcome of a binomial variate: the acceptance probability was set to 1 if $A_{V}(\mathrm{Gal})>1 \mathrm{mag}$, to $2 / 3$ otherwise.

To model the effect of the second bias, we preliminarily compiled a list of the ground facilities that mostly contributed to measuring GRB redshifts. For each declination, we considered the visibility from each location by calculating the fraction of time that a generic source at the given declination spends at $>+30^{\circ}$ above the local horizon. This threshold value matches average observational constraints. We then averaged this value over the full set of locations, weighting by the multiplicity of the number of telescopes at a given location (e.g. in La Palma there are several telescopes that provided GRB redshift measurements). We have therefore come up with a probability of having $z$ measured as a function of declination. This bias was incorporated in the MC simulations as a further criterion for a random position to be accepted, depending on the outcome of a binomial variate: the acceptance probability was set as one minus the probability of having $z$ measured for that declination.

We also applied method 2 by weighting each sky pixel by the product of the sky exposure and the two above probabilities. We then calculated the mean value for each moment and the corresponding uncertainties as in Sect. 3.2 under the same assumptions. The results are reported in Table 2. Both methods gave fully consistent results as was the case for the other samples.

It is noteworthy that the deviation from isotropy of the Galactic quadrupole moment $\left(\sin ^{2} b-1 / 3\right)$ tapers off from $-2.8 \sigma$ to $-1.8 \sigma$ when observational biases are accounted for. Likewise, the equatorial quadrupole moment $\left(\sin ^{2} \delta-1 / 3\right)$ decreases from $+2.3 \sigma$ to $+1.7 \sigma$.

We also considered the Galactic dust correction alone and found that it accounts for $\sim 90 \%$ of the total change in the expected Galactic quadrupole moment, so it dominates over the correction for the locations of ground telescopes.

\subsection{Constraining the fraction of a possible Galactic population}

The Galactic quadrupole moment $\left(\sin ^{2} b-1 / 3\right)$ of unknown$z$ events is marginally deviating from isotropy: this holds both for the FREDs (S2) and, to somewhat lower extent for the nonFREDs (SnFnZ). This would imply an excess of events at low Galactic latitudes. Taken at face value, when one overlooks the impact of the observational biases discussed in Sect. 4.4 and plainly assumes that this is mostly due to the presence of a Galactic population, following T12, one can estimate its fraction within the sample of unknown- $z$ FREDs.

We did so by generating 100 synthetic samples with as many events as in S2, a fraction of which was obtained from an isotropic distribution, and the remaining fraction was obtained from a random selection of a sample of 334 known Galactic sources from the BAT catalogue by Cusumano et al. $(2010)^{2}$. Both populations were selected compatibly with the exposure map.

By varying every time the fraction of the Galactic component, we ended up with the best match between observations and simulations. This turned into an estimated fraction of Galactic intruders in S2 of $f=(27 \pm 10) \%(1 \sigma)$, similar to T12's conclusions. In practice, given that the evidence for anisotropy is only marginal, we provided a $90 \%$ confidence upper limit of $f<40 \%$ by demanding that at least $90 \%$ of the synthetic samples had a non-zero quadrupole moment at $>3 \sigma$.

However, neglecting the observational biases has the effect of overestimating the weight of the possible Galactic population. We therefore repeated the same test by accounting for those biases. Following the procedure of Sect. 4.4, we came up with a more realistic estimate of $f=(19 \pm 11) \%$ with a $90 \%$ upper limit of $f<34 \%$.

\section{Discussion}

We found no evidence for a significant dipole moment in the different FRED samples observed by Swift. However, we point out that this does not clash with T12's claim: in contrast with what they assert, $\cos b$ is by no means a dipole, since a dipole moment is the cosine of the angle between a generic direction and a fixed direction in the sky, not a plane. Thus, the disagreement is in the claim, but not in the results, which do agree. In particular, significant dipole moment was found neither along the Galactic poles $(\sin b)$ nor towards the Galactic centre $(\cos b \cos l)$.

In contrast, and in agreement with T12, we found that the Galactic quadrupole moment $\left(\sin ^{2} b-1 / 3\right)$ of unknown- $z$ events marginally deviates from isotropy, implying an excess of events at low Galactic latitudes.

However, before giving credence to the possible existence of this relatively abundant disguised Galactic community, one must carefully evaluate the impact of observational biases other than the non-uniform exposure map. That not just FREDs, but all GRBs with unknown $z$ tend to cluster towards the Galactic plane, likely suggests that the impact of the process of measuring $z$ might be far from negligible. This possibility appears to be corroborated by the absence of non-zero quadrupole moments in the entire sample of BAT events (STot), in spite of its having the best statistical sensitivity. Low Galactic latitude GRBs are notoriously disfavoured in their optical counterpart detection

2 We made use of known sources just to mimic in a credible way
what the distribution of a Galactic population should look like. Clearly,
such possible unrecognised Galactic sources must obviously be different from the known Galactic sources. 
and consequent $z$ measurement because of the strong Galactic dust extinction. Moreover, our result that unknown- $z$ events tend to cluster around the celestial poles $\left(|\delta|>60^{\circ}\right)$, where groundbased follow-up observations are less probable (Fynbo et al. 2009), lends further support to the z-related observational bias explanation. In the light of these considerations, we modelled the impact of both effects in shaping the unknown- $z$ sample $S 2$ by preliminarily studying how the measurement of $z$ is hampered by the amount of Galactic dust content for the entire sample of Swift GRBs. Furthermore, we modelled the probability for a generic source at a given declination to be followed up spectroscopically by estimating its visibility from the most frequently used ground facilities. As a result, we found that the deviation from isotropy of the Galactic quadrupole moment decreased from $-2.8 \sigma$ to $-1.8 \sigma$, thus deflating the body of evidence of a significant contamination due to a disguised population of Galactic transients.

We delved deeper into this matter by estimating the contaminating fraction of a Galactic population that would yield the same Galactic quadrupole moment as that observed in the sample of unknown- $z$ FREDs. When the observational biases are properly accounted for, we estimate $f=(19 \pm 11) \%(1 \sigma)$ with a $90 \%$ upper limit of $f<34 \%$.

To gain further insight, we examined the spectral and temporal properties of the $\gamma$-ray prompt emission along with the X-ray afterglow of the $\mathrm{S} 2$ sample, as a function of the Galactic position, and compared their distributions with those derived for a sample of cosmological GRBs with measured $z$. Specifically, we estimated the rise and the decay times of the $\gamma$-ray profiles as well as the photon index in the $15-150 \mathrm{keV}$ range of the spectrum extracted over the $T_{90}$ interval. The $\mathrm{X}$-ray afterglow properties were taken from the classifications provided by Swift-XRT catalogues (Evans et al. 2009; Margutti et al. 2013) and from the online Leicester catalogue ${ }^{3}$ for recent GRBs. We did not find evidence for any of these observables for a different distribution between S2 and cosmological GRBs.

It is noteworthy that the fraction of short duration GRBs in S2 is less than $7 \%(8 / 119)$, i.e. far from enough to account for the deviation from isotropy that we observe. Several investigations on past short and very short GRB catalogues brought evidence for anisotropy (Balazs et al. 1998; Magliocchetti et al. 2003; Vavrek et al. 2008; Cline et al. 2003, 2005). However, the low fraction of short GRBs in our Swift samples rules them out as the main cause for the marginally observed anisotropy.

\section{Conclusions}

Triggered by previous results, we searched for the possible presence of disguised Galactic transients in the catalogue of GRBs detected by Swift-BAT up to September 2014. We focussed on FRED GRBs, given that their light curves are often observed in a broad range of Galactic high-energy transients. The search was based on the statistical analysis of the sky distribution both with reference to our Galaxy and in a way that is independent of a reference-system. While no deviation from isotropy was found in the dipole moment, we found marginal $(2.8 \sigma)$ evidence for a non-zero Galactic quadrupole moment for FREDs with unknown redshift $z$, suggesting a clustering at low Galactic latitudes, in particular, towards the celestial poles $\left(|\delta|>60^{\circ}\right)$. If this possible clustering on the Galactic plane is due the presence of an unidentified Galactic population, we constrain its fraction to $f=(27 \pm 10) \%$. However, non-FRED GRBs with unknown $z$ also show a similar behaviour, although with lower statistical significance $(2.1 \sigma)$. If one drops the unknown- $z$ condition, this evidence disappears. It is known that there are observational biases that disfavour the measurement of $z$ : the large dust extinction towards the Galactic plane, along with the lower probability of ground-based observations of polar $\left(|\delta|>70^{\circ}\right)$ events, can contribute to enhance the degree of anisotropy. Once these biases are accounted for, the evidence for a clustering near the Galactic plane decreases from $\sim 3 \sigma$ to $\sim 2 \sigma$, thus weakening the claim for a Galactic contamination. Furthermore, comparing the properties of the $\gamma$-ray prompt emission, as well as of the X-ray afterglow with those of GRBs with measured $z$, did not reveal any statistical difference. Hence, our results suggest marginal evidence for the presence of an unrecognised Galactic population among the GRBs with unknown redshift.

In conclusion, when we account for $z$-measurement-related biases, the fraction of a possible disguised Galactic population of high-energy transients among Swift-BAT GRBs with unknown redshift is estimated to be $f=(19 \pm 11) \%$, with a $90 \%$-confidence upper limit of $f<34 \%$.

Acknowledgements. We are grateful to the referee for comments that improved the paper. We thank G. Cusumano, S. Dichiara, F. Frontera, C. Koen, P. Rosati, for useful discussions. PRIN MIUR project on "Gamma Ray Bursts: from progenitors to physics of the prompt emission process", P. I. F. Frontera (Prot. 2009 ERC3HT) is acknowledged. This work made use of data supplied by the UK Swift Science Data Centre at the University of Leicester.

\section{References}

Balazs, L. G., Meszaros, A., \& Horvath, I. 1998, A\&A, 339, 1

Barthelmy, S. D., Barbier, L. M., Cummings, J. R., et al. 2005, Space Sci. Rev., 120,143

Bernui, A., Ferreira, I. S., \& Wuensche, C. A. 2008, ApJ, 673, 968

Briggs, M. S. 1993, ApJ, 407, 126

Briggs, M. S., Paciesas, W. S., Pendleton, G. N., et al. 1996, ApJ, 459, 40

Burrows, D. N., Hill, J. E., Nousek, J. A., et al. 2005, Space Sci. Rev., 120, 165

Castro-Tirado, A. J., de Ugarte Postigo, A., Gorosabel, J., et al. 2008, Nature, 455,506

Cline, D. B., Matthey, C., \& Otwinowski, S. 2003, Astropart. Phys., 18, 531

Cline, D. B., Czerny, B., Matthey, C., Janiuk, A., \& Otwinowski, S. 2005, ApJ, 633, L73

Cucchiara, A., Levan, A. J., Fox, D. B., et al. 2011, ApJ, 736, 7

Cusumano, G., La Parola, V., Segreto, A., et al. 2010, A\&A, 524, A64

Evans, P. A., Beardmore, A. P., Page, K. L., et al. 2009, MNRAS, 397, 1177

Fynbo, J. P. U., Jakobsson, P., Prochaska, J. X., et al. 2009, ApJS, 185, 526

Guidorzi, C. 2015, Astron. Comput., 10, 54

Hartmann, D., \& Blumenthal, G. R. 1989, ApJ, 342, 521

Hartmann, D., \& Epstein, R. I. 1989, ApJ, 346, 960

Kasliwal, M. M., Cenko, S. B., Kulkarni, S. R., et al. 2008, ApJ, 678, 1127

Lewin, W. H. G., van Paradijs, J., \& Taam, R. E. 1993, Space Sci. Rev., 62, 223

Magliocchetti, M., Ghirlanda, G., \& Celotti, A. 2003, MNRAS, 343, 255

Margutti, R., Zaninoni, E., Bernardini, M. G., et al. 2013, MNRAS, 428, 729

Mereghetti, S. 2008, A\&ARv, 15, 225

Mészáros, P., \& Gehrels, N. 2012, RA\&A, 12, 1139

Norris, J. P., Nemiroff, R. J., Bonnell, J. T., et al. 1996, ApJ, 459, 393

Pietka, M., Fender, R. P., \& Keane, E. F. 2015, MNRAS, 446, 3687

Remillard, R. A., \& McClintock, J. E. 2006, ARA\&A, 44, 49

Schlafly, E. F., \& Finkbeiner, D. P. 2011, ApJ, 737, 103

Segreto, A., Cusumano, G., Ferrigno, C., et al. 2010, A\&A, 510, A47

Stefanescu, A., Kanbach, G., Słowikowska, A., et al. 2008, Nature, 455, 503

Tanvir, N. R., Chapman, R., Levan, A. J., \& Priddey, R. S. 2005, Nature, 438, 991

Tegmark, M. 1996, ApJ, 470, L81

Tello, J. C., Castro-Tirado, A. J., Gorosabel, J., et al. 2012, A\&A, 548, L7

Vavrek, R., Balázs, L. G., Mészáros, A., Horváth, I., \& Bagoly, Z. 2008, MNRAS, 391, 1741

\footnotetext{
3 http://www.swift.ac.uk/
} 